\title{
Identidad y legitimidad de la cooperación al desarrollo: El debate sobre la relación de la AOD con la pobreza y la desigualdad internacional
}

KOLDO UNCETA SATRUSTEGUI

Instituto Hegoa, UNIVERSIDAD DEL PAÍS VASCO (UPV/EHU), ESPAÑA. E-mail: koldo.unceta@ehu.es

JORGE GUTIÉRREZ-GOIRIA

Instituto Hegoa, UNIVERSIDAD DEL PAÍS VASCO (UPV/EHU), ESPAÑA. E-mail: jorge.gutierrez@ehu.es

\section{RESUMEN}

Los debates sobre la Ayuda Oficial al Desarrollo, su eficacia e interés no son nuevos, aunque la actual crisis haya aumentado la presión crítica en este sentido. En este contexto, es importante repasar el papel de la AOD tradicionalmente asociado a la disminución de la pobreza y de la desigualdad internacional- para analizar tanto su relación con los cambios habidos en estas variables, como la posible influencia de todo ello en su propia identidad. Para ello, el trabajo examina en primer término la evolución del discurso sobre dichas cuestiones, para después ahondar en la escasa evidencia existente sobre la incidencia de la AOD en la evolución de las mismas durante el período 1990-2010.

Palabras clave: Ayuda Oficial al Desarrollo, pobreza, desigualdad.

\section{Identity and Legitimacy of Development Cooperation: The Debate on the Relationship between ODA, Poverty and International Inequality}

\begin{abstract}
Discussions on Official Development Assistance, its effectiveness and interest are not new, but the current crisis has increased the critical pressure in this regard. In this context it is important to review the role of ODA -traditionally linked to the reduction of poverty and inequality- to analyze its relationship with the changes in these variables, and also the potential influence of this on its own identity. To do this, the work first examines the evolution of the discourse on these issues, and then analyzes the limited evidence on the impact of ODA in the evolution of these questions during the period 1990-2010.
\end{abstract}

Keywords: Official Development Assistance, Poverty, Inequality.

Clasificación JEL: F35, I32

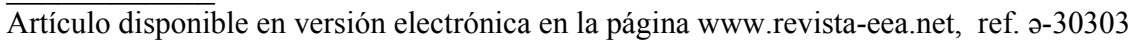




\section{INTRODUCCIÓN}

La crisis financiera que afecta especialmente a algunos países de la OCDE ha tenido en ciertos casos una repercusión directa en los flujos de AOD y en el compromiso de algunos gobiernos respecto a los mismos. Sin embargo, los debates sobre la eficacia de la cooperación y sobre el interés de mantener la misma vienen de más atrás. Las dudas sobre los objetivos y la orientación de la AOD se han venido manifestando de diversa manera a lo largo de las dos últimas décadas, condicionando tanto la denominada Agenda del desarrollo, como la cuantía y las condiciones de los flujos de aquella. En este contexto, las cuestiones relativas a la eficacia de la cooperación han compartido la atención con los análisis referidos a sus objetivos, si bien ambos tipos de aproximaciones se han planteado casi siempre de manera paralela e inconexa.

Por un lado, y refiriéndonos al tema de la eficacia, desde mediados de la década de 1990 y al calor de lo que se vino a llamar la "fatiga de la ayuda", el debate sobre el impacto real de la misma adquirió una creciente relevancia, tanto en ámbitos académicos como políticos y sociales ${ }^{1}$. En 1998 el Banco Mundial publicó Assessing Aid: what works, what doesn't, and why, trabajo que marcó buena parte de los estudios posteriores sobre la eficacia de la cooperación y su relación con la necesidad de entornos favorables, buenas políticas, condicionalidades, etc. Sin embargo, con el comienzo del nuevo siglo, y tras las reuniones de Roma y Paris, buena parte de las preocupaciones sobre la eficacia se trasladaron a la propia cadena de la ayuda. El resultado fue la conformación de dos espacios diferentes para evaluar la AOD: unos centrados en el posible impacto de la misma sobre determinados agregados macroeconómicos, como el ahorro, la inversión, o el crecimiento; y otros orientados a estudiar los principios que debían regir el propio funcionamiento de los programas de cooperación y ayuda y la relación entre donantes y receptores. Esta ausencia de claridad sobre el propio objeto de análisis ha influido sin duda en las confusas controversias de los últimos años sobre el impacto y la eficacia de la cooperación al desarrollo, algunas de las cuales -como la polémica mantenida por Paul Easterly y Jeffrey Sachs- llegaron a alcanzar cierto eco mediático ${ }^{2}$.

Por otra parte, y de forma paralela, se ha llevado a cabo una amplia discusión sobre los propios objetivos y sobre la orientación de la cooperación, lo que ha venido a llamarse el debate sobre la Agenda. Ya en plena crisis de la AOD en los años 90, la Cumbre de Copenhague fue escenario de diversos intentos para priorizar la lucha contra la pobreza y los aspectos sociales del desarrollo. Con posterioridad la puesta en escena de los ODM (Objetivos del Milenio) vino a consagrar una orientación centrada en una agenda de mínimos en la que la po-

\footnotetext{
${ }^{1}$ Un trabajo de referencia en este aspecto fue el de Cassen (1994).

${ }^{2}$ Ver a este respecto Sotillo (2011).
} 
breza extrema tendría un protagonismo especial. Esta posición ha sido no obstante discutida desde diversas perspectivas señalándose la necesidad de tener en cuenta la multidimensionalidad de los problemas en presencia y su relación con asuntos más amplios que condicionan el desarrollo y la propia lucha contra la pobreza (Easterly, 2008; Kabeer, 2010). Además, la desatención -rallando en el menosprecio- registrada hacia el octavo de los Objetivos del Milenio -que afecta a la necesidad de cambios de mayor calado en las relaciones financieras o comerciales- ha servido para confirmar algunos diagnósticos más pesimistas sobre el paulatino reduccionismo en la agenda de la cooperación

Severino y Ray (2009) plantean por su parte que la AOD ha sufrido importantes cambios que se derivan de sus nuevos objetivos, de la proliferación de agentes que intervienen en la misma, o de la expansión de los instrumentos financieros utilizados, apuntando la necesidad de abandonar incluso el propio término -AOD- sustituyéndolo por el de Política Financiera Global (GPF en sus siglas inglesas), basada en tres aspectos principales: la convergencia entre las economías del Norte y del Sur; un mejor acceso a servicios esenciales en el mundo, y la provisión de Bienes Públicos Globales como la protección medioambiental o la salud internacional.

En estas circunstancias, puede observarse una creciente confusión en el debate sobre el papel de la AOD y el futuro de la cooperación al desarrollo, sin que exista un consenso suficiente sobre las referencias que deberían ser tenidas en cuenta a la hora de discutir tanto sobre su eficacia pasada como sobre el papel que pueda desempeñar en adelante. Lo cierto es que la profundidad de los cambios acaecidos en las últimas décadas, y la diversidad de preocupaciones y puntos de vista presentes en el debate ha conducido a la AOD a lo que algunos autores como Sobhan (2006) han llamado una "crisis existencial", que afecta a la consideración de su supremacía, eficacia y legitimidad como instrumento de ayuda hacia los países más pobres, lo cual se ve afectado asimismo por la menor importancia relativa de la AOD tiene desde 1990 en el conjunto de las transferencias internacionales de capital hacia dichos países (Sobhan, 2006, Unceta et al., 2010). En este orden de cosas, Sörensen (2010) analiza la crisis del modelo de cooperación tal como fue concebido por los países occidentales tras la segunda guerra mundial, comparándolo con el actual modelo de relaciones establecido por China y otros países asiáticos frente a los países de África o América Latina.

Autores como Easterly (2008) enfatizan la necesidad de abandonar los grandes discursos y los planteamientos de tipo general -entre los que sitúa los ODM y las ideas propuestas en los últimos años desde los principales organismos internacionales-, para centrar la atención en los problemas concretos, cuya amplitud y complejidad ha ido incrementándose en los últimos años. Sin embargo, la distancia que separa las discusiones sobre la Agenda del Desarrollo y los 
Objetivos de la Cooperación por un lado, y los debates sobre la eficacia por otro, complica la delimitación del debate. Como señala Van der Been (2011), las ideas sobre los objetivos y propósitos de la política de ayuda dan forma a sus formulaciones e implementación, generándose una situación desconcertante, porque no es evidente a priori cuál debe ser es el objetivo de la asistencia oficial para el desarrollo.

Las anteriores consideraciones afectan no solo al debate académico sobre el papel de la AOD sino que inciden directamente en la consideración de la misma por parte de la ciudadanía. Tras el fuerte impulso ético de la misma durante sus inicios, en los últimos años ha ido ganando importancia el debate técnico en el seno de Organismos Internacionales, Agencias de Desarrollo y ONGD, sin que la opinión pública alcance a conocer cuáles son realmente los objetivos que se persiguen, ni en qué medida los esfuerzos realizados están contribuyendo a su consecución. Los dos grandes anhelos que dieron origen a la cooperación al desarrollo tras la segunda guerra mundial -la lucha contra la pobreza y la desigualdad internacional, concretados en lo que se denominó el cierre de la brecha Norte/Sur- siguen de alguna manera presentes en el imaginario colectivo, pese a la evolución que el tratamiento de los mismos ha tenido en el debate académico y político sobre la AOD.

Estas dos grandes cuestiones -el aumento o disminución de la pobreza y de la llamada brecha Norte/Sur- han sido objeto de atención a lo largo de los últimos años. Por una parte, han proliferado los trabajos que, de una u otra manera, han centrado su atención en los avances o retrocesos registrados en la reducción de la pobreza extrema, tal como ha sido planteada en los ODM. También, y aunque sin plantear una conexión directa con dichos objetivos, recientemente se han registrado notables aportaciones al debate sobre la desigualdad internacional, planteándose nuevos diagnósticos sobre la misma, algunos de los cuales vienen a cuestionar parte de las visiones tradicionales sobre la denominada brecha Norte/Sur. Sin embargo, ni unas ni otras aportaciones han planteado la existencia de vínculos manifiestos entre los cambios habidos en la incidencia de la pobreza extrema -o en las tendencias de las desigualdades internacionales- y la evolución seguida por la AOD durante todo este tiempo. Y ello, pese a los intensos debates registrados o a los llamamientos efectuados por algunos organismos internacionales sobre la necesidad perentoria de incrementar de la AOD para cumplir con algunos objetivos propuestos, caso de los $\mathrm{ODM}^{3}$. No obstante, y más allá de estas y otras excepciones, apenas se establecen conexiones evidentes entre los avances o retrocesos en los objetivos, y el papel que desempeña

\footnotetext{
${ }^{3}$ El Informe del PNUD sobre el Desarrollo Humano de 2005 lanzaba una alarma en esa dirección señalando que el cumplimiento de los ODM sería imposible si no se duplicaba la AOD. Por su parte, el Informe Social Watch de 2007 planteaba que los ODM se cumplirían sólo 100 años después, y ello como consecuencia de la ausencia de compromisos suficientes sobre la AOD.
} 
o puede desempeñar la AOD. Pareciera que nos encontráramos ante dos debates paralelos, que apenas se cruzan, pese a la intensidad de los mismos.

En este marco, el presente texto se centra en analizar esta aparente paradoja. Si los escasos logros que en términos macro fueron diagnosticados en los años 90 en materia de pobreza y desigualdad internacional sirvieron para cuestionar abiertamente la cooperación al desarrollo, dando origen a una amplia literatura sobre el tema, en la actualidad no parece estar ocurriendo lo contrario. Los principales organismos internacionales se hacen eco de importantes avances en la lucha contra la pobreza, pero nadie parece vincular los mismos con el papel de la AOD. Así las cosas, la crisis de identidad de esta última sigue aumentando a medida que crecen las dudas tanto sobre la incidencia que está teniendo en la evolución de algunos problemas, como sobre el papel que podría jugar -y no está jugando- de cara a enfrentar otros.

Para nuestro propósito, partimos de examinar en primer lugar la paulatina transformación registrada en el discurso sobre los principales objetivos del desarrollo desde las formulaciones iniciales planteadas en los inicios de la AOD hasta hoy. En segundo término, presentamos un resumen de los principales resultados planteados por diversos autores y organismos sobre la evolución reciente de la pobreza y las desigualdades internacionales. A continuación analizamos -a la luz de los datos de AOD disponibles- si puede establecerse alguna relación concluyente entre ambos planos del discurso. Finalmente, y en base a todo lo anterior, presentamos algunas conclusiones sobre el momento por el que atraviesa la cooperación al desarrollo y la posible crisis de legitimidad que le afecta.

\section{LA DESIGUALDAD INTERNACIONAL Y LA LUCHA CONTRA LA POBREZA EN EL DISCURSO DE LA COOPERACIÓN}

Como es bien conocido, los inicios de la cooperación al desarrollo estuvieron marcados por la necesidad de hacer frente a la nueva realidad surgida tras los procesos de descolonización iniciados después de la segunda guerra mundial, y la evidencia de dos mundos contrapuestos, Norte y Sur, caracterizados por las grandes diferencias existentes entre ellos, y por la presencia en el segundo de importantes bolsas de pobreza y privación humana. En ese contexto, la cooperación y los flujos de AOD se asociaron desde el primer momento al objetivo de "disminuir" o "cerrar" la brecha Norte/Sur, mediante la superación de los problemas de pobreza presentes en los que fueron denominados "países en desarrollo". De esa manera, y aunque se tratara en realidad de cuestiones conceptualmente diferentes, la lucha contra la pobreza y la necesidad de enfrentar la desigualdad internacional fueron presentados como partes de un mismo objetivo. 


\subsection{Las desigualdades internacionales y la disminución de la brecha Norte/Sur}

De la importancia concedida a la denominada brecha Norte/Sur en el discurso de la cooperación al desarrollo dan buena muestra las primeras palabras del Informe Pearson, publicado en 1969, tras la constitución -a instancias del Banco Mundial- de la Comisión de Desarrollo Internacional, encargada de examinar los avances y retrocesos registrados tras dos décadas de esfuerzos de cooperación para el desarrollo. Al inicio de dicho informe, reproduciendo literalmente un texto de Naciones Unidas de 1951, se señalaba: "La amplia brecha existente entre los países desarrollados y los países en desarrollo constituye el principal problema de nuestro tiempo" "4.

A lo largo de varias décadas, el argumento de la desigualdad entre países constituyó una de las cuestiones centrales en la literatura sobre el desarrollo y la cooperación y, de alguna manera, la AOD fue concebida como una herramienta mediante la que los países ricos debían contribuir al desarrollo de los países pobres, acercando a éstos a los estándares de aquellos y cerrando la brecha existente entre unos y otros. Estas ideas estuvieron presentes en las principales declaraciones internacionales así como en los documentos que sirvieron de base a la proclamación de la primera y la segunda Décadas para el desarrollo. La propia declaración sobre el establecimiento de un Nuevo Orden Económico Internacional aprobada por las Naciones Unidas en 1974 se hacía eco de estas cuestiones señalando que la disparidad entre los países desarrollados y los países en desarrollo continuaba aumentando, y que la cooperación internacional constituía, más que nunca, una herramienta fundamental para superar dicha situación (Naciones Unidas, 1974).

La insistencia en la necesidad de cerrar la brecha Norte/Sur y en plantearla como objetivo central de la cooperación se fue consolidando de forma paralela a los análisis que apuntaban a un creciente aumento de la misma, tanto en términos absolutos como relativos. Al término de la primera década para el desarrollo, y haciendo balance de la misma, las NN. UU. llamaban la atención sobre el ensanchamiento de la distancia entre unos y otros países ${ }^{5}$. Algo después, en 1977, el Banco Mundial publicaba un Informe en el que, repasando 25 años de políticas de desarrollo, se alertaba sobre el gran aumento de la brecha Norte/Sur y la necesidad de hacer frente a la misma (Morawetz, 1977). Por su parte, el Informe de la Comisión Brandt, dado a conocer en 1981, subrayaba el hecho de que los llamados países del Norte comprendían sólo una cuarta parte de la po-

\footnotetext{
${ }^{4}$ UNESCO (1970). El Informe de NN. UU. al que se hace referencia se titulaba "Measures for the Economic Development and Under-Developed Countries", y fue publicado en 1951.

${ }^{5}$ NN.UU.: http://www.nationsencyclopedia.com/United-Nations/Economic-and-Social-Development-FIRST-UN-DEVELOPMENT-DECADE.html
} 
blación mundial pero acaparaban cuatro quintas partes del ingreso, en tanto los países considerados del Sur, que suponían tres cuartas partes de la población del mundo, solo disponían de una quinta parte del ingreso total. Por otro lado, además de los Informes de Naciones Unidas o del Banco Mundial, diversos autores, como Bairoch (1973) o Seligson (1984) llamaron también la atención sobre el problema de la brecha Norte/Sur relacionándola con los retos de la cooperación.

Con posterioridad, la preocupación por la desigualdad internacional y el papel que la cooperación al desarrollo pudiera jugar para paliarla siguió presente en el discurso de gran parte de las agencias de cooperación, si bien en un marco de creciente disputa con las ideas que defendían la mayor eficacia de la globalización y el mercado de cara a ese propósito (Baghwati, 2005; Sala i Martín, 2001 etc. $)^{6}$. El propio Banco Mundial sugería en 1994 que los considerados países en desarrollo -tomados en conjunto- estaban registrando tasas de crecimiento económico más elevadas que las de los países industrializados, lo que podría suponer un acortamiento de la brecha, punto de vista que fue contestado por diversos trabajos, como el de Broad y Merlhon (1996) que enfatizaba la necesidad de desagregar los datos y considerar el negativo impacto que en la mayoría de los países considerados había tenido la década de 1980, llegando a la conclusión de que no se podía hablar en modo alguno de mejoras en la brecha Norte-Sur. Esta situación favoreció que desde algunos sectores se continuara defendiendo el papel de la AOD en la disminución de las desigualdades entre unos y otros países. Como apuntaba Alonso (2001) el ensanchamiento de la distancia entre países ricos y pobres durante ese período dejaba poco margen de confianza en el mercado de cara a dicho objetivo, haciendo que la ayuda al desarrollo continuara percibiéndose como un resorte necesario para promover la equidad.

Sin embargo, en los últimos años, las cuestiones relativas a la desigualdad internacional han pasado a un segundo o tercer plano en los debates sobre la cooperación al desarrollo, a la vez que se fijaba el objetivo en la lucha contra la pobreza extrema. Lo cierto es que desde comienzos de siglo las referencias a la llamada brecha Norte/Sur han ido poco a poco desapareciendo en el discurso sobre la AOD. A nuestro modo de ver, ello está relacionado con dos asuntos principales. Por un lado con la menor significación de los términos Norte y Sur en la actualidad, al menos en la manera en que los mismos habían sido utilizados para describir una panorama mundial polarizado en torno a estas dos realidades. Y por otra parte, dicho giro ha tenido que ver asimismo con el cambio de

\footnotetext{
${ }^{6}$ Ya desde los años noventa comenzó a proliferar una amplia literatura sobre la cuestión de la convergencia o divergencia entre países, centrada sobre todo en el análisis de las condiciones que permitían unas tasas de crecimiento superiores en unos países que en otros. Ver a este respecto Sachs y Warner (1995).
} 
discurso sobre la cuestión de la pobreza que, desde los años 90 y muy especialmente desde la promulgación de los ODM, ha centrado su atención en la extrema pobreza, cuestión de la que nos ocupamos en el siguiente apartado.

\subsection{La cooperación al desarrollo y la lucha contra la pobreza}

Al igual que la superación de la brecha Norte/Sur, la lucha contra la pobreza fue planteada desde los inicios como objetivo central de la cooperación al desarrollo. Partiendo de los diagnósticos sobre los círculos viciosos de la pobreza realizados por varios de los pioneros de la economía del desarrollo como Rosenstein-Rodan, Nurkse, o Myrdal, se impuso la idea de la necesidad de la aportación de capital y tecnología externas para poder romper dichos círculos y emprender el camino del desarrollo. De esa forma, junto a la idea de emular el camino seguido por los países considerados más prósperos, el objetivo de eliminar o paliar la pobreza se convirtió en elemento motriz de las políticas de cooperación, asociándolo directamente a los avances que pudieran registrarse en materia de crecimiento económico.

Como es sabido, la escasa o nula evidencia registrada en esta relación durante las primeras décadas de la cooperación al desarrollo (ya que las tasas de crecimiento agregado no se habían correspondido con reducciones equivalentes -ni tampoco aproximadas- en las tasas de pobreza) vino a cuestionar la eficacia de las políticas seguidas ${ }^{7}$ y a plantear la necesidad de una nueva estrategia, que se conoció como enfoque de las Necesidades Básicas (NN.BB.). El propio Informe Pearson antes mencionado, consideró insignificantes los progresos realizados en este tema durante las primeras dos décadas de desarrollo, planteando la necesidad de redoblar los esfuerzos para poder reducir la pobreza en el mundo.

Pese al énfasis puesto en las Necesidades Básicas y en la necesidad de priorizar las mismas, los discursos sobre la cooperación no dejaron de lado las cuestiones relativas a las desigualdades internacionales, al tiempo que los análisis de la pobreza en términos relativos continuaban formando parte de los debates sobre el desarrollo. Buena parte de la literatura sobre los umbrales de pobreza ${ }^{8}$ siguió insistiendo en la idea de la inclusión social y la necesidad de tener como referencia los patrones o modelo de vida existentes en unas y otras sociedades. Algunos de los textos más relevantes sobre las NN.BB. se hicieron eco asimismo de las distintas posibilidades de entender las mismas, planteándolas como mínimos vitales o bien de acuerdo a lo que cada sociedad podía considerar un nivel de vida decente (Stewart, 1985; Streeten, 1981).

\footnotetext{
${ }^{7}$ El debate afectó al propio Banco Mundial en el que bajo la presidencia de McNamara se impulsaron diversos estudios sobre la incidencia real del crecimiento económico en la absorción de la pobreza. Algunos de ellos fueron publicados en Chenery et al. (1974).

${ }^{8}$ Ver entre otros, Sen (1983), Kakwani (1986), o Atkinson (1987).
} 
Sin embargo, tras la crisis sufrida por la AOD en los años noventa y las dudas planteadas sobre la eficacia y los resultados de la misma ${ }^{9}$, el discurso oficial pasó a centrarse en la consideración de la pobreza absoluta y en la definición de unos objetivos mínimos en torno a los cuales centrar los esfuerzos. Dicho proceso se inició con la publicación del Informe del Banco Mundial de 1990, dedicado precisamente al tema de la pobreza. En dicho informe se definió esta última como la incapacidad de alcanzar un nivel de vida mínimo en base a dos criterios: por una parte, considerando el gasto necesario para acceder a un estándar mínimo de nutrición y otras necesidades muy básicas, y por otro reconociendo la existencia de una cantidad variable capaz de reflejar el coste de la participación en la vida diaria de unas y otras sociedades (World Bank, 1990). Sin embargo, esta doble aproximación a la pobreza en términos tanto absolutos como relativos sería abandonada progresivamente para poner el foco de atención en el coste de los aspectos más básicos, lo cual se justificó en buena medida por razones operativas, aunque la apuesta por encarar prioritariamente la lucha contra la pobreza absoluta no estuvo exenta de controversias, especialmente tras la Cumbre de Copenhague de $1995^{10}$.

Con el comienzo del nuevo siglo, la definición de los Objetivos del Milenio representó la consolidación del enfoque basado en la pobreza absoluta y, dentro de éste, en la prioridad concedida a la extrema pobreza. Ravallion, uno de los economistas más influyentes del Banco Mundial, defendía el umbral de pobreza absoluta (1 dólar al día) basando su argumento en su sencillez y operatividad (Ravallion, 2000) ${ }^{11}$. Sin embargo, trabajos realizados desde diferentes perspectivas vinieron a cuestionar la metodología empleada para evaluar la pobreza (Deaton, 2003; Reddy y Pogge, 2002; Vandemoortelee, 2002,) y el propio PNUD -en su Informe sobre del Desarrollo Humano del año 2003- se hacía eco de estos asuntos, apuntando la existencia de graves problemas en la medida y en la interpretación de la pobreza, y subrayando la ausencia de un consenso suficiente sobre el tema ${ }^{12}$.

El nuevo enfoque daría lugar a su vez a un nuevo discurso sobre la efectividad de la AOD a la hora de reducir la pobreza, basada en algunos modelos e

\footnotetext{
${ }^{9}$ Un buen resumen de las incertidumbres sobre la AOD en los años 90 y su contextualización en las distintas fases por las que ha venido atravesando la cooperación puede verse en Browne (1997).

${ }^{10}$ Para un examen del proceso de consolidación de la lucha contra la pobreza como aspecto central de la estrategia de cooperación ver Vandemoortele (2009).

${ }^{11}$ Dicho umbral había sido establecido por el Banco Mundial en 1990, si bien posteriormente se elevó a 1.08\$ por día en PPP correspondientes a 1993, de acuerdo con las propuestas de Chen y Ravallion (2000).

${ }^{12}$ A todo ello es necesario añadir el debate sobre la relación entre la distinta importancia de la pobreza de ingreso en unos y otros contextos y la compleja relación entre los aspectos monetarios y no monetarios de la pobreza. Ver a este respecto Stewart, Saith y Harris-White (2007).
} 
ideas como las propuestas por Collier y Dollar (2000, 2001), que estarían en la base de lo que el propio banco llamaría un nuevo consenso internacional sobre el papel de la AOD y su contribución a la reducción de aquella (World Bank, 2002), basado en la cuestión de las condicionalidades y en considerarla, en todo caso, un complemento de las buenas políticas. Paralelamente se intensificarían los esfuerzos tendentes a defender tanto el enfoque centrado en la pobreza extrema como los umbrales establecidos ${ }^{13}$.

\section{POBREZA Y DESIGUALDAD INTERNACIONAL: EVOLUCIÓN Y ANÁLISIS RECIENTES}

Más allá de los debates sobre el enfoque y las prioridades de la cooperación internacional, durante los últimos años han proliferado los estudios dirigidos a examinar los avances o retrocesos habidos tanto en lo que afecta a la pobreza como en lo que tiene que ver con la desigualdad internacional. En las próximas líneas nos detendremos brevemente sobre estas dos cuestiones.

\subsection{Evolución de los análisis y los diagnósticos sobre la desigualdad internacional}

Como hemos comentado más atrás, hasta finales de los años 90 buena parte de los análisis y trabajos publicados consideraban que la desigualdad entre los países en términos de ingreso por habitante había continuado ensanchándose en el contexto del nuevo proceso de globalización abierto en los años $80^{14}$, en línea por otra parte con las tendencias que habían caracterizado buena parte del siglo XX. La propia Asamblea General de las Naciones Unidas se hacía eco de esta cuestión al señalar en 1988 que la brecha entre países ricos y pobres se había ampliado, y constituía la principal amenaza para el desarrollo y para la paz (Jo1ly, 2005). Sin embargo, autores como Sachs y Warner, señalaron que la mayor parte de los estudios presentados sobre esta materia a finales de los 80 y principios de los 90 eran excesivamente pesimistas sosteniendo que, bajo ciertas condiciones -entre las que incluían diversos criterios de apertura al exterior, eficiencia económica, derechos humanos y otros-, los países pobres tenían un

\footnotetext{
${ }^{13}$ A partir de 2008 el nuevo umbral quedaría establecido en $1,25 \$$ diarios, tomando como base un nuevo trabajo Ravalion, Chen, y Sangraula (2008). En este texto, los propios autores reconocen que el umbral que se propone es conservador pero argumentan que acotar el problema -centrándolo en los más pobres de entre los pobres- contribuye a una mejor orientación de las políticas.

${ }^{14}$ En un conocido texto sobre las ideas y las prácticas de las NN. UU. en torno a la cuestión del desarrollo, se señalaba a este respecto que "la corriente principal del discurso sobre la globalización en los años 90 enfatizaba el potencial beneficio de ésta en términos de crecimiento sostenido y prosperidad, pero desconsidera el agudo incremento de las desigualdades dentro y entre los países" (Emmerij et al., 2001).
} 
importante margen para paliar la brecha que les separaba de los más ricos (Sachs y Warner, 1995).

También los informes de los principales organismos internacionales se ocuparon del aumento de la brecha Norte/Sur en las décadas finales del siglo XX. Así, el Informe del PNUD sobre Desarrollo Humano correspondiente a 1999 señalaba que las diferencias de renta entre los países más pobres y los más ricos habían seguido creciendo y que si, en 1960, el $20 \%$ de la población de los países más ricos poseía 30 veces más ingresos que el 20\% más pobre, en 1997 tenía 74 veces más ${ }^{15}$. Por su parte el Banco Mundial apuntaba en 2001 que si en 1960, el PIB per cápita de los veinte países más ricos era 18 veces mayor que el de los veinte países más pobres, en 1995 era ya 37 veces mayor, lo que según el Banco venía a indicar que "en los últimos 40 años la desigualdad de la renta entre los países ha aumentado de forma dramática" (World Bank, 2000).

Sin embargo, desde la última década los diagnósticos se han ido modificando, planteándose otras perspectivas en la consideración de las desigualdades internacionales, así como nuevos datos referidos a los indicadores tradicionalmente utilizados, es decir la comparación entre el PIB/hab. de unos y otros países. Respecto del primero de estos asuntos, diversos autores han venido proponiendo la necesidad de superar la visión de las desigualdades mundiales basada en el ingreso per cápita de cada país completándola con un diagnóstico más integral que contemple tanto la importancia relativa de cada país (teniendo en cuenta su población), como la cuestión referida a las desigualdades internas dentro de los mismos, asunto este último de especial relevancia en la actuali$\mathrm{dad}^{16}$. En este marco, resulta útil la distinción empleada por Milanovic (2005), y seguida entre otros por Sutcliffe (2011), para medir la desigualdad a nivel mun$\operatorname{dia}^{17}$.

En esta línea, tal como muestra Suttcliffe (2011), puede observarse que el índice de Gini resultante de suponer un mundo poblado por un habitante de cada país (enfoque de tipo I), con unos ingresos equivalentes a su PIBpc, ha ido disminuyendo entre 1980 y 2009, lo que daría idea de una distribución del PIB mundial progresivamente más equitativa. También en el caso del enfoque del Tipo II, y desde aproximaciones algo diferentes, se observa una tendencia si-

\footnotetext{
${ }^{15} \mathrm{El}$ Informe apuntaba asimismo que la distancia entre el promedio de ingreso del país más rico y el más pobre era de 3 a 1 en 1820 , de 11 a 1 en 1913, de 35 a 1 en 1950 , 44 a 1 en 1973 y 72 a 1 en 1992 (PNUD, 1999)

${ }^{16}$ Refiriéndose a los EE. UU. Fujuyama señala que si bien el 1\% de las familias (las más ricas) ingresaba el 9\% del PIB en 1974, en 2007 ingresaba ya el 23,5\% del PIB, a la vez que apunta que la mediana del ingreso lleva estancada en dicho país desde 1970 (Fujuyama, 2012).

${ }^{17}$ Dicha metodología distingue entre desigualdad de Tipo I (entre países, considerando el PIB per cápita y a cada país como una unidad), Tipo II (ponderando la cifra anterior según el tamañonúmero de habitantes) y Tipo III (que mide la distribución entre personas).
} 
milar, que podría indicar una convergencia en términos de distribución de renta a escala internacional (entre países). Sin embargo, estas dos medidas de la desigualdad, aún cuando pudieran mostrar algunos avances, presentan un enfoque muy centrado en el Estado-nación, y desvían la atención sobre la cuestión clave en términos de desigualdad, que es la distribución entre las personas y familias. Así, si se pasa a medir la desigualdad entre personas (Tipo III), encontramos que, pese a la convergencia en términos de los PIB nacionales (totales o per cápita), las diferencias de renta entre los individuos están aumentando a nivel mundial.

Lo anterior resulta sumamente significativo e incide en la consideración de las brechas a escala mundial. Tal como explica Sutcliffe (2011) con base en los trabajos de Milanovic, las estimaciones indican que, en efecto, la desigualdad global de toda la población mundial (tipo III) subió entre 1988 y 2008, pese a la caída en los tipos I o II. Ello plantea la necesidad de una nueva mirada sobre la desigualdad que vaya más allá del marco de los estados nación y considere los efectos de la globalización sobre este fenómeno. En este sentido, la desigualdad como problema asociado a los retos el desarrollo podría haber perdido buena parte de su significación como categoría analítica cuando se considera en términos internacionales (tipo I) ${ }^{18}$, pero representar sin embargo un reto y una amenaza considerable si se contempla en su dimensión global

\subsection{Pobreza}

En el caso de la pobreza, los diagnósticos oficiales sobre esta cuestión han venido incidiendo durante los últimos años en la idea de una mejora permanente de la situación ${ }^{19}$. Ya en 2002, el Banco Mundial anunciaba una importante reducción en el número de personas con menos de 1 dólar al día, que habría variado desde el 32\% de la población mundial en 1990 al 25\% en 1999 (Vandermoortele, 2002). Desde entonces, esta idea se ha venido repitiendo con tintes cada vez más optimistas, especialmente en los últimos años en los que un buen número de trabajos han venido señalando que la pobreza tiende a disminuir, o al menos a estabilizarse, debido sobre todo al fuerte ritmo de crecimiento económico experimentado en algunos países del continente asiático. En efecto, la mayor parte de los estudios realizados durante la pasada década coinciden en subrayar el efecto positivo de los cambios en el sur y el este de Asia sobre la evolución de la pobreza en el mundo. China ha sido mencionada de forma recu-

${ }^{18}$ Ello no obstante, puede identificarse un grupo de países (PMA) para los que también la brecha tipo I ha ido aumentando (Jolly, 2005). Dicho fenómeno ya había sido observado con anterioridad por Reynolds (1986), referido al grupo de países con peores resultados en términos de crecimiento

${ }^{19}$ Una visión de conjunto sobre los diagnósticos en torno a la pobreza llevados a cabo en los primeros años de la década de 2000 puede verse en Unceta (2007). 
rrente a este respecto (Sachs, 2005; Stiglitz 2006; Vandemoortele, 2002), aunque algunos autores (Dollar, 2006) han subrayado también la incidencia del crecimiento económico registrado en otros países como India ${ }^{20}$, Bangladesh, o Vietnam. Ello vendría a indicar que la gran importancia de todos estos países en términos demográficos podría haber condicionado buena parte de los diagnósticos globales sobre la pobreza en el mundo.

En el lado opuesto de los análisis ha venido situándose África Subsahariana. Buena parte de los diagnósticos sobre la evolución de la pobreza han venido apuntando hasta hace poco que en este continente es en donde el problema era más agudo, o incluso endémico, mientras en el resto del mundo se trataría de un fenómeno en vías de solución. Dollar (2006) señalaba a este respecto que si bien dos tercios de la población en situación de extrema pobreza vivían en Asia, las tasas de crecimiento de este continente harían que en poco tiempo la pobreza se acabara concentrando en África. Stiglitz (2006), sin embargo, venía a subrayar que la excepción era más bien China y no tanto África, dado que durante los últimos tiempos la pobreza se había incrementado en la mayor parte de los países en desarrollo, si bien coincidía a la hora de señalar que África representaba el mayor fracaso en la lucha contra la pobreza. Para Sachs (2005), mientras en África la pobreza está aumentando tanto en números absolutos como en porcentaje de población, en las regiones asiáticas había disminuido en ambos aspectos, posición que coincidía con la expuesta por el propio Banco Mundial en su informe de 2006 según el cual mientras los mayores éxitos se habían dado se forma casi exclusiva en Asia, casi todos los incrementos de la pobreza se habían registrado en África Subsahariana (World Bank, 2005).

Sin embargo, resulta obligado relacionar los análisis y diagnósticos llevados a cabo sobre la evolución de la pobreza con el tipo de indicadores y de umbrales utilizados para medirla, cuestión a la que nos hemos referido con anterioridad. Así, Chen y Ravallion (2004), Dollar (2006), o Sachs (2005) reconocían que, si bien el porcentaje de personas con menos de 1 dólar al día -los denominados extremadamente pobres- se había reducido, el de quienes vivían con menos de 2 dólares al día -los llamados moderadamente pobres- había aumentado. Por otra parte, y además de la cuestión de los umbrales, otro asunto de gran relevancia es el que tiene que ver con la utilización de porcentajes o de cifras absolutas. En este sentido, desde la formulación de los ODM, se viene trabajando en términos porcentuales, lo que no permite visualizar el número de personas que se encuentran en situación de extrema pobreza en el mundo. Así, por ejemplo, el informe del Banco Mundial (Chen y Ravallion, 2012) señala que entre 1990 y

${ }^{20}$ El caso de la India es significativo pues las estadísticas del Banco Mundial presentan importantes lagunas en la información referida a este país. Datt y Ravallion (2002) apuntaban a una leve mejoría en los años 90, pero los datos sobre la evolución de la pobreza en los años 2000 son escasos y parciales. 
2008 prácticamente se había reducido a la mitad el porcentaje de personas pobres (de $43,1 \%$ a $22,4 \%$ ), pero el número total de personas en situación de pobreza (dado el incremento de población) pasó de 1.225 a 1.116 millones, lo que supone un descenso inferior al 9\%, mucho menos esperanzador. Los propios Chen y Ravallion (2007) reconocen que la evolución es distinta si se tiene en cuanta uno u otro aspecto, ya que la reducción porcentual de las personas en situación de extrema pobreza no había ido acompañada de una reducción paralela del número de pobres en términos absolutos.

Más allá de esta cuestión, los problemas metodológicos afectan a un variado abanico de cuestiones. Una de ellas tiene que ver con las dificultades existentes para la recogida y tratamiento de estas muestras y encuestas, problema reconocido por el propio Banco Mundial. Aunque se han realizado indudables avances, los datos recogidos son insuficientes, y en el mejor de los casos se dispone de los mismos para períodos de 3 en 3 años -y ello de forma reciente, lo que dificulta estudiar la evolución y sus posibles determinantes ${ }^{21}$. Por su parte, Broad y Cavanagh (2009) señalan que las cifras aportadas por Sachs y otros autores sobre la evolución de la pobreza son confusas y subrayan la inconveniencia de utilizar los mismos umbrales al margen de tratarse de economías rurales de subsistencia o de pobres urbanos, lo que les lleva a concluir que la aproximación oficial al tema de la pobreza se basa en estadísticas engañosas que llevan a propuestas erróneas.

Sea como fuere, el optimismo en torno a la reducción de la pobreza no ha ido sino aumentando en los últimos tiempos. En marzo de 2012, una nota del Banco Mundial, suscrita por Chen y Ravallion, actualizaba los datos de la institución señalando que, por vez primera vez desde 1981, el porcentaje de personas viviendo con menos de 1,25 dólares al día había disminuido en todas las regiones del mundo, incluida África Subsahariana, lo que sirvió para que algunos medios hablaran de cumplimiento de los ODM antes de la fecha estable$\mathrm{cida}^{22}$. En esta misma línea, y de forma aún más optimista, un estudio de Chandi y Gertz (2011), con una metodología algo diferente, estimaba que el porcentaje de personas en extrema pobreza en 2015 , más que reducirse a la mitad, quedaría en la cuarta parte del ratio inicial, situándose por debajo del $10 \%$ de la población mundial. Sin embargo, estos diagnósticos sobre la reducción de la pobreza han sido matizados por algunos autores que plantean la necesidad de complementar estos análisis con los cambios habidos en términos de riesgos, vulnera-

${ }^{21}$ A todo ello habría que añadir las consideraciones planteadas desde distintos sectores sobre el tratamiento de la pobreza exclusivamente en términos de ingreso, cuando existe un creciente consenso sobre el carácter multidimensional de la misma y los datos de varios de los últimos informes del PNUD muestran el empeoramiento de la situación en algunos aspectos.

${ }^{22}$ Ver The New York Times 6 marzo 2012 (http://www.nytimes.com/2012/03/07/world/extremepoverty-down-despite-recession-world-bank-data-show.html) 
bilidad e incremento de las desigualdades. Este último asunto podría resultar un elemento crítico en el medio plazo ya que como señala Kanbur (2010) la reducción de la pobreza asociada a un aumento de la desigualdad constituye un fenómeno propio de algunas situaciones con una elevada tasa de crecimiento.

\section{EL PAPEL DE LA AOD EN LA DESIGUALDAD Y EN LA LUCHA CONTRA LA POBREZA}

Llegados a este punto queremos fijar nuestra atención en la posible relación existente entre los avances mencionados en el apartado anterior sobre la evolución de la pobreza y de la desigualdad internacional (tipo I), y la trayectoria seguida por los flujos de la AOD, tanto en términos globales, como en su dirección hacia unos u otros tipos de países. Se trata de valorar si puede establecerse alguna relación de causalidad entre ambos tipos de fenómenos o si, por el contrario, no hay evidencias sobre ello.

\subsection{Desigualdades internacionales y flujos de AOD}

Por lo que se refiere a la posible disminución de las brechas entre países mencionada en el punto anterior, los estudios relativos a la misma que han sido mencionados no contemplan la cuestión de la AOD ni la posible incidencia de la misma. De manera general, la mayor parte de las explicaciones sobre la disminución de las brechas entre países tienden a subrayar los efectos de la globalización y las nuevas condiciones de la economía mundial como aspecto determinante, sin que otros elementos ajenos al mercado merezcan apenas consideración. Dollar (2006), por ejemplo, subraya la existencia de una mayor integración y convergencia en la economía mundial, lo que supone una menor distancia en términos de ingreso per cápita, lo que sin embargo es atribuido a la creciente apertura exterior y a la mayor integración comercial con el resto del mundo. En cualquier caso, la constatación del inicio de una cierta convergencia en términos estrictamente de PIB/hab., y su relación con la globalización y la difuminación de los espacios económicos estato-nacionales no puede ocultar la persistencia de una fuerte controversia sobre el alcance real y las consecuencias de la mencionada globalización. Mientras algunos señalan dicha convergencia como la prueba de sus bondades, otros enfatizan el aumento de las desigualdades globales, el incremento de las tensiones sociales y medioambientales, y la emergencia de nuevos y más graves problemas de desarrollo.

Por nuestra parte, hemos tomado los datos del propio Banco Mundial para compararlos con la evolución de los flujos de la AOD. En las Figuras 1 y 2 puede observarse, por una parte, la evolución del ingreso per cápita de distintas 
regiones $^{23}$ como proporción del correspondiente a los países de la OCDE; y por otro lado, la evolución de los flujos de AOD hacia esas mismas regiones. En la primera de dichas figuras destaca el Este de Asia como zona del mundo en la que la brecha -medida en ingreso per cápita- se ha reducido más respecto de los países de la OCDE, mientras que, en el extremo opuesto, África Subsahariana destaca por el estancamiento, o incluso ligero retroceso sufrido en ese mismo aspecto. En la segunda de las figuras, podemos ver sin embargo que -dejando de lado el errático comportamiento de los flujos hacia África del Norte y Medio Oriente $^{24}$-, África Subsahariana es la región del mundo en la que más ha crecido la AOD recibida, mientras que en el Este de Asia la misma ha permanecido estancada.

En la Figura 3 pueden observarse dichos fenómenos de manera conjunta para los dos casos significativos seleccionados. El creciente flujo de AOD per cápita en el caso de África Subsahariana contrasta con el estancamiento en el caso asiático (ver la evolución de las líneas punteadas en relación al eje derecho de AOD per cápita). Sin embargo, la evolución de la brecha muestra una clara disminución en el caso asiático que contrasta con su mantenimiento en África (ver las líneas continuas en relación al eje izquierdo, que mide el porcentaje de PIBpc de la región en relación al de la OCDE). Esto nos permite concluir que, en términos regionales, no existe evidencia alguna que relacione los flujos de AOD con las variaciones en el diferencial de ingreso per cápita.

Figura 1

Evolución de la brecha de ingreso OCDE-otras regiones 1990-2010.

Porcentaje sobre PIBpc OCDE (PPA, US\$ corrientes)

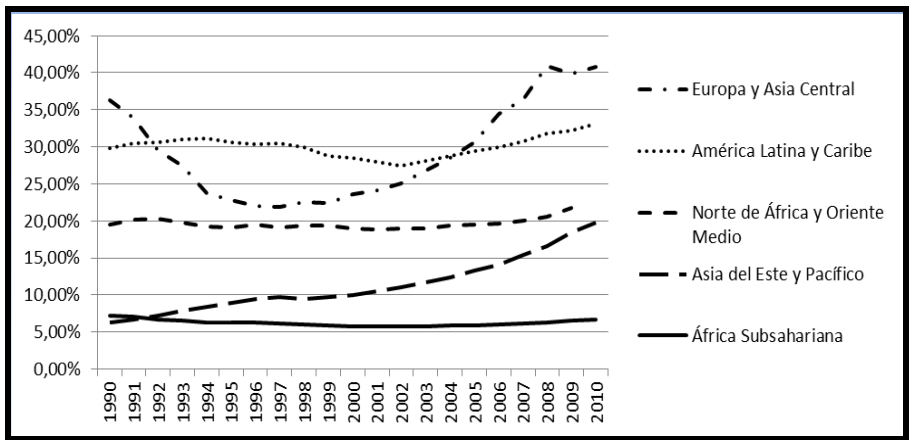

Fuente: Elaboración propia en base a datos del Banco Mundial.

${ }^{23}$ A efectos del estudio se han seleccionado los datos referidos a los países clasificados como "en desarrollo" por el propio Banco Mundial en cada región.

${ }^{24}$ Las cifras se hallan muy condicionadas por el impacto contable de fondos relacionados con los conflictos de Oriente próximo y Afganistán. 
Figura 2

AOD recibida per cápita por zonas 1990-2010 (US\$ corrientes)

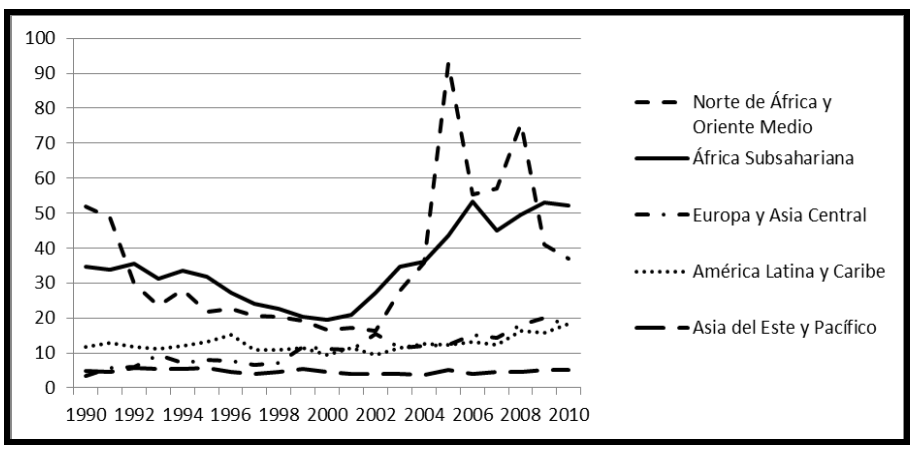

Fuente: Elaboración propia en base a datos del Banco Mundial.

Figura 3

Evolución de la brecha de ingreso respecto a la OCDE y AOD recibida per cápita en África SS y Asia del Este y Pacífico

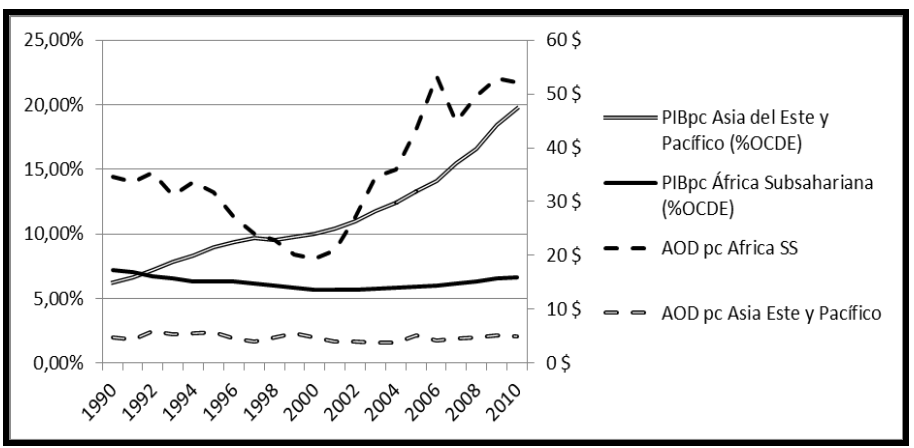

Fuente: Elaboración propia en base a datos del Banco Mundial.

Finalmente, en la Figura 4 se muestra la ausencia de relación entre ambas cuestiones partiendo de los datos disponibles para un total de 113 países. En el eje de abscisas se ha medido la disminución (en positivo) o aumento (en negativo) de la brecha relativa (en porcentaje) entre el PIBpc de cada país y el correspondiente para el conjunto de la OCDE entre los años 1990 y 2010. Por su parte, en el de ordenadas, se ha calculado la AOD per cápita promedio para el período. Cada punto representa un país de renta media o baja de los que se dispone de ambos tipos de datos para el inicio y final del período. La figura muestra una nube dispersa y en la que no puede apreciarse ningún efecto positivo entre la mayor AOD recibida y un avance en términos de disminución de la brecha. Es destacable que en 46 de los 113 países recogidos la brecha de hecho 
haya aumentado, incluso en estos términos relativos y en PPA que se han considerado.

Figura 4

Evolución de la brecha de ingreso respecto a la OCDE y AOD recibida per cápita para 113 países $(1990-2010)^{25}$

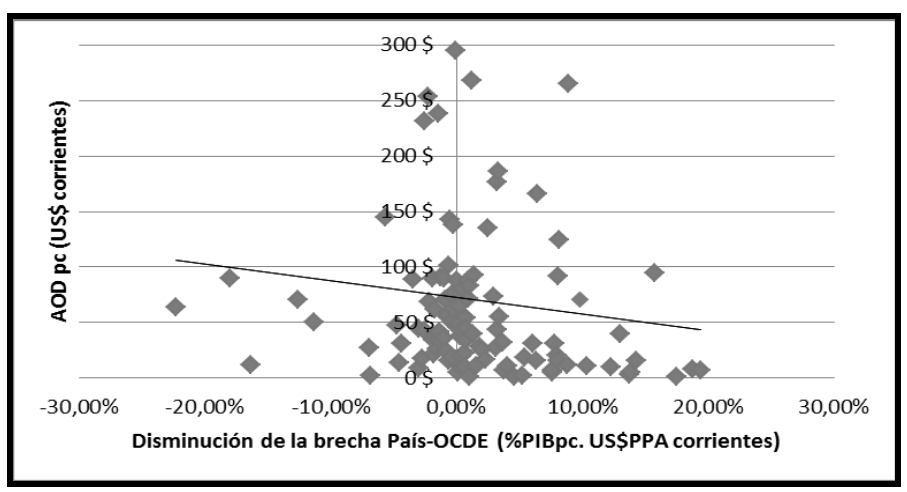

Fuente: Elaboración propia en base a datos del Banco Mundial.

\subsection{Evolución de la pobreza y de los flujos de AOD}

A diferencia de lo ocurrido con el tema de las desigualdades internacionales y la brecha Norte-Sur, durante la última década se han llevado a cabo diversos estudios sobre el papel de la AOD en la evolución de la pobreza. En efecto, tras los cuestionamientos habidos a mediados de los años 90 sobre la incidencia real de la AOD, a comienzos de la década de 2000 otros trabajos cuestionaron, matizaron o arrojaron nuevas perspectivas sobre este asunto, o plantearon el debate sobre las condiciones en las que la AOD podía ser más eficaz para reducir la pobreza (Burnside y Dollar, 2000; Collier y Dollar, 2001; Hansen y Tarp, 2001). Con posterioridad, en los últimos años se han planteado nuevos análisis y perspectivas sobre estos temas. Por un lado, han proliferado los estudios sobre los costes de financiación y las necesidades de AOD asociadas a las metas de lucha contra la pobreza contenidas en los ODM y a las dudas sobre el cumplimiento de las mismas (Devarajan et al., 2002; UN Millennium Project, 2005). Por su parte, algunos autores han insistido en cuestiones de método relacionadas con las preguntas a realizar y con los aspectos que deben ser examinados ${ }^{26}$ para

\footnotetext{
${ }^{25}$ Nota: se ha excluido de la figura el caso extremo de Micronesia, con una AOD pc muy elevada, al objeto de mejorar la visualización de la figura, sin que esto afecte a la interpretación de la misma.

${ }^{26}$ Los temas a debate son muchos y abarcan desde la relación entre el corto y el medio plazo, las cuestiones de la eficiencia, la volatilidad, los tipos y condiciones de la ayuda, las diferentes dimensiones de la pobreza y las relaciones que se establecen entre ellas, etc.
} 
poder establecer un juicio al respecto (Easterly, 2006; Riddell, 2007). Otros trabajos (Clemens et al., 2007) han cuestionado tanto la propia formulación de los ODM como las relaciones establecidas entre su cumplimiento y el incremento de los flujos de AOD. E incluso personas tan cualificadas en la definición de los ODM y las metas de lucha contra la pobreza como Vandemoortele (2009) han discutido la excesiva dependencia de la ayuda externa que, desde algunos círculos, se ha querido dar al cumplimiento de dichos objetivos, subrayando la escasa atención prestada a otras cuestiones que, como las desigualdades internas, pueden hacer peligrar ese cumplimiento.

Pese a todo ello, y acotando el debate a la incidencia que la AOD haya podido tener en la reducción porcentual de la pobreza absoluta, apenas se han aportado evidencias al respecto. Por una parte, existen numerosas dificultades asociadas a la carencia de datos fiables y homologables, tanto en lo relativo a la AOD como en lo que se refiere a la reducción de la pobreza. Y por otro lado, parece cada vez más claro que las condiciones de cada país -o incluso de cada zona dentro de un país- resultan determinantes para favorecer o limitar el posible impacto de la AOD en la reducción de la pobreza, lo que dificulta la obtención de conclusiones generales sobre el tema (Schabbel, 2007).

Por nuestra parte, y partiendo de la misma base de datos del Banco Mundial, hemos examinado las tendencias generales observadas en el comportamiento de ambas variables. La Figura 5 muestra la evolución general (en términos agregados) tanto de la pobreza absoluta como de la AOD, que muestra una aparente coincidencia entre la disminución del porcentaje de personas que viven por debajo del umbral de pobreza considerado y el incremento habido en los flujos de cooperación.

Figura 5

AOD (millones de dólares constantes 2009) y porcentaje de personas con menos de $1,25 \$$ al día en el mundo (1990-2008)

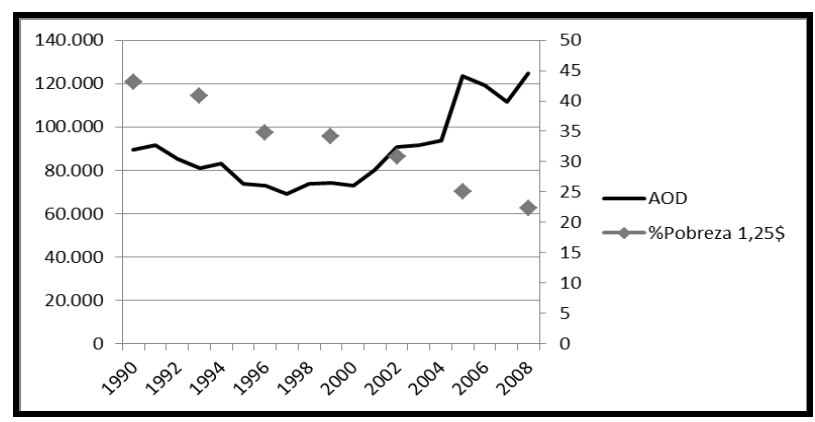

Fuente: Elaboración propia en base a datos del Banco Mundial. 
Ahora bien, como ya se ha señalado anteriormente, la mayor parte de la literatura sobre la evolución de la pobreza ha considerado China y África Subsahariana como los máximos exponentes del éxito -en un caso- o del fracaso -en el otro- en el tema de la reducción de la pobreza. Por ello, hemos centrado nuestra atención en ambos casos, con los resultados que pueden verse en las Figuras 6 y 7.

Figura 6

AOD recibida (millones de dólares constantes 2009) y porcentaje de personas con menos de 1,25\$ al día en China (1990-2008)

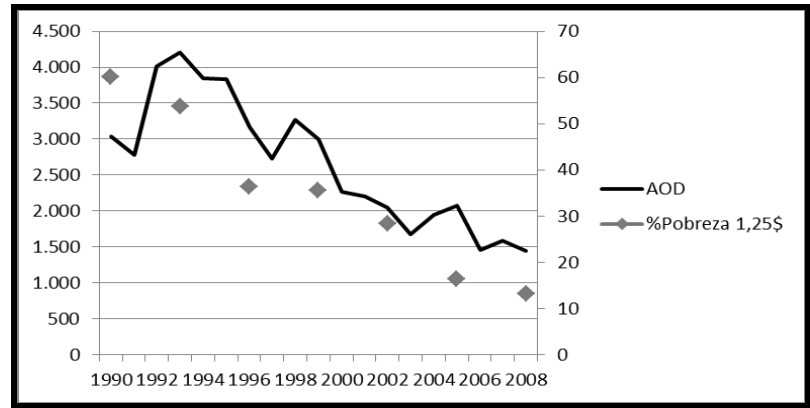

Fuente: Elaboración propia en base a datos del Banco Mundial.

Figura $n^{\circ} 7$

AOD recibida (millones de dólares constantes 2009) y porcentaje de personas con menos de 1,25\$ al día en África Subsahariana (1990-2008)

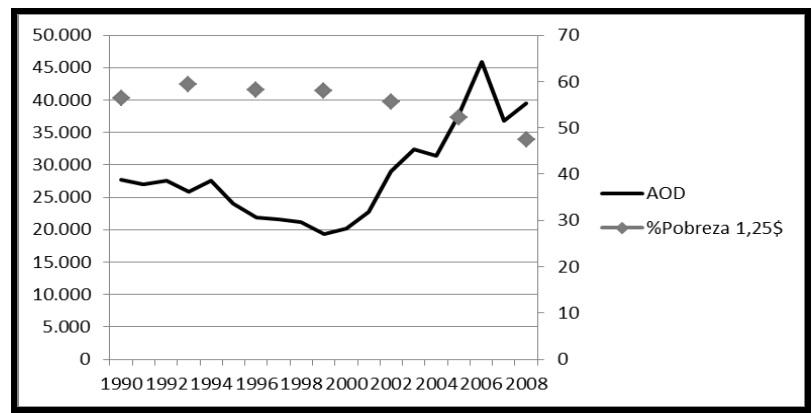

Fuente: Elaboración propia en base a datos del Banco Mundial.

Como puede observarse, las cifras disponibles sobre reducción de pobreza y AOD no permiten establecer en el caso de China una relación como la esperada al respecto, ya que la disminución de la primera habría ido de la mano de una 
reducción paralela de la $\mathrm{AOD}^{27}$. Por su parte, las cifras relativas a África Subsahariana indican una tendencia inicialmente decreciente en los flujos de la AOD hacia dicha región, sin que se observen apenas cambios en términos de pobreza. El posterior aumento de AOD (se duplica entre 1999 y 2008), no se acompaña de reducciones comparables en términos de pobreza. Considerando globalmente el período, resulta difícil con estos datos concluir que haya una relación coherente entre ambos fenómenos.

Más allá de estos resultados, y a diferencia de lo realizado en el apartado anterior (4.1), la escasez de datos disponibles no permite presentar una figura de dispersión relacionando la disminución en la incidencia de la pobreza y la AOD per cápita recibida por distintos países. Es preciso señalar a este respecto que los datos del Banco Mundial para el período contemplado (1990-2008) solo permitirían incluir 8 casos en que se disponga de datos de incidencia de la pobreza en los años inicial y final ${ }^{28}$. Sea como fuere, en dichos casos tampoco se podía observar relación alguna entre disminución de la pobreza y AOD per cápita recibida.

\section{CONCLUSIONES}

Llegados a este punto plantearemos algunas conclusiones sobre el tema tratado.

A lo largo de las dos últimas décadas las discusiones en torno a la AOD han girado en torno a dos grandes temas. Por un lado, el discurso sobre los objetivos de la misma, centrándolos cada vez más en lo que hemos llamado una agenda de mínimos, vinculada a la Ayuda Humanitaria y a los Objetivos del Milenio. Y por otra parte, el debate sobre la eficacia de la Ayuda que ha basculado entre la atención a los efectos de la cooperación en términos macroeconómicos y la consideración de las relaciones donantes-receptores dentro de la cadena de la ayuda, concretados en los principios de París.

La mayor parte de los análisis sobre la mencionada agenda de mínimos, centrados en la evolución de la pobreza, han insistido en la positiva evolución de la misma en términos relativos, esto es, considerando el porcentaje -que no el número- de personas que viven bajo el umbral considerado (1,25 dólares PPA). Al mismo tiempo, y aunque el tema ha desaparecido prácticamente de la

${ }^{27}$ Easterly (2006:354) ironiza a este respecto señalando que "el Banco Mundial está supuestamente salvando gente de la pobreza en China con un presupuesto muy limitado. A partir de 2002, 563 millones de dólares al año, o lo que es lo mismo alrededor de la décima parte de un centavo por persona y día".

${ }^{28}$ La metodología de análisis Banco Mundial, a efectos de paliar esta escasez de datos, plantea el cálculo de valores de referencia cada 3 años, lo que permite que, con solo tener un valor en el trienio (bien en el año de referencia, el anterior o el posterior) se pueda extrapolar o estimar un valor, aumentando así las posibilidades de comparación. 
agenda, los análisis sobre la desigualdad internacional consideran que durante la última década se ha invertido por vez primera la tendencia histórica de más de un siglo, y que la brecha entre países, medida tradicionalmente en PIB/hab. ha comenzado a decrecer, a la vez que se iniciaba un proceso de convergencia.

Los análisis sobre la evolución de estas dos variables han servido para que algunos sectores reivindiquen el protagonismo del mercado y discutan la propia existencia de los organismos y los programas internacionales relacionados con la cooperación. Pero más allá de la utilización interesada de los datos para lanzar mensajes de una indudable carga ideológica, lo cierto es que los mencionados cambios han venido a cuestionar los fundamentos históricos de la ayuda al desarrollo y los objetivos que, referidos a la misma, han sido transmitidos a la ciudadanía a lo largo de las últimas décadas. Los actuales retos del desarrollo requieren probablemente de nuevos y más cuantiosos esfuerzos en materia de cooperación, pero éstos deberán basarse en otros fundamentos y asociarse a nuevas metas e instrumentos.

Es preciso tener en cuenta que muchos de los análisis más triunfalistas relativos a la reducción de la pobreza absoluta dejan de lado matices muy importantes relacionados con la metodología utilizada y que cuestionan parte de los progresos señalados, como los umbrales de pobreza considerados, o la utilización de variaciones porcentuales frente a número de personas. En otros casos se omiten cuestiones de gran importancia como son las relativas a la incidencia de algunos países (especialmente China e India) en las cifras globales. Y, finalmente pero no por ello menos importante, destaca la ausencia de análisis sobre la emergencia de nuevas y graves amenazas para el desarrollo, como el gran incremento de las desigualdades internas o los crecientes problemas medioambientales que requerirían nuevas propuestas e instrumentos de cooperación.

Pero más allá de estas limitaciones, llama la atención la ausencia de estudios que examinen la relación entre los supuestos avances mencionados y la evolución de los flujos de AOD. Tras los estudios elaborados y los debates habidos a mediados de la década pasada sobre las necesidades de financiación de los ODM y el papel de la cooperación de cara a su cumplimiento, lo cierto es que el reciente triunfalismo mantenido a este respecto en algunos círculos oficiales no se ha visto acompañado de análisis sobre la incidencia que la AOD haya podido tener en los avances mostrados. Curiosamente, si en los años 90 los escasos avances en la reducción de la pobreza y la desigualdad internacional sirvieron de base para cuestionar la eficacia y el papel de la cooperación, en la actualidad el alarde de los logros obtenidos no se ha visto acompañado de ningún tipo de reivindicación sobre el posible rol de la AOD en todo ello.

En este contexto, nuestro análisis ha buscado establecer algún tipo de relación entre ambos fenómenos (reducción de la pobreza o de las brechas internacionales por un lado y variaciones en los flujos de AOD por otro), sin que los 
resultados obtenidos permitan establecer ningún vínculo al respecto. En efecto, los datos oficiales existentes no permiten constatar ninguna evidencia de que la AOD tenga alguna relación con las variaciones observadas en las tasas de reducción de la pobreza o en la disminución de las desigualdades entre países.

Todo lo anterior plantea, a nuestro modo de ver, algunos interrogantes sobre el papel presente y futuro de la cooperación. Por un lado, el discurso sobre la misma se ha ido reduciendo paulatinamente, limitándose poco a poco las metas a perseguir. La consolidación de los ODM y su cumplimiento como piedra de toque a la hora de analizar la eficacia de la cooperación es una buena muestra de ello, pese al abandono en la práctica del octavo de dichos objetivos y el olvido de su incumplimiento en los análisis más triunfalistas sobre el tema. Pero, al mismo tiempo, la ausencia de evidencias sobre el papel que la AOD haya podido tener en los logros exhibidos proyecta algunas dudas sobre su futuro.

A nuestro modo de ver, los avances habidos en algunos países en la reducción de la pobreza absoluta, o las variaciones registradas en la desigualdad internacional de tipo I no deberían representar las únicas -ni siquiera las principales- referencias a la hora de evaluar los avances en materia de desarrollo en un contexto como el actual en el que las brechas sociales y medioambientales siguen creciendo, en el que las incertidumbres y amenazas para la seguridad humana son mayores que nunca. Se trata de una situación en la que los esfuerzos de cooperación internacional, con nuevas propuestas e instrumentos, deberían redoblarse, a la vez que se debería avanzar en la imprescindible asociación internacional para el desarrollo señalada en el octavo de los ODM. Sin embargo, el vaciamiento habido en los últimos años en el ámbito de los discursos y los análisis sobre la cooperación, unido a su creciente tecnificación, han erosionado gravemente los fundamentos de la misma así como su propia legitimación social. En estas circunstancias, consideramos que el discurso, la lógica y los valores asociados a la cooperación al desarrollo se encuentran seriamente dañados, frente al avance de las aproximaciones al tema que defienden el papel del mercado como única referencia.

El espejismo producido por algunos pequeños logros -amplificados y presentados como grandes triunfos desde algunos círculos oficiales- puede estar ocultando la gravedad de otras dimensiones del desarrollo no menos importantes que, además de amenazar la estabilidad de dichos logros, representan retos de gran envergadura que requerirán nuevos y diferentes esfuerzos de cooperación internacional. Pero ello requeriría una nueva perspectiva en los estudios y análisis sobre la misma, capaz de superar el reduccionismo de las últimas décadas. 


\section{REFERENCIAS BIBLIOGRÁFICAS}

ALONSO, JOSÉ ANTONIO (2001). "Nuevas direcciones en la política de ayuda al desarrollo" en Revista de Economía Mundial $n^{\circ}$ 5, pp. 11-45.

ATKINSON, ANTHONY B. (1987). "On the measurement of poverty" en Econometrica, V. 55, n 4, pp. 749-764.

BHAGWATI, JAGDISH (2005). En defensa de la globalización. Barcelona: Debate.

BAIROCH, PAUL (1973). El Tercer Mundo en la encrucijada. Madrid: Alianza Editorial.

BROAD, ROBIN Y MELHORN, CHRISTINA (1996). "Whither the North: South Gap?" en Third World Quarterly, Vol. 17, No. 1, pp. 7-17.

BROAD, ROBIN Y CAVANAGH, JOHN (2009). Development Redefined. How the market met its match. London: Paradigm Publishers.

BROWNE, STEPHEN (1997): "The Rise and Fall of Development Aid". World Institute for Development Economics Research Working Papers, n 143.

BURNSIDE, CRAIG, Y DOLLAR, DAVID (2000). "Aid, Policies, and Growth" en The American Economic Review, Vol. 90, № 4, pp. 847-868.

CASSEN, ROBERT (1994). Does Aid Work? Oxford: Clarendon Press.

CHANDY, LAURENCE Y GERTZ, GEOFFREY (2011). "Poverty in Numbers: The Changing State of Global Poverty from 2005 to 2015". Brookings Global Views Policy Briefs, 2011-01.

CHEN, SHAOHUA Y RAVALLION, MARTIN (2000). "How did the World's Poorest Fare in the 1990s?" World Bank. Policy Research Working Paper, $\mathrm{n}^{\circ}$ 2409. Washington

CHEN SHAOHUA Y RAVALLION, MARTIN (2004). "How have the world poorest fared since the early 1980s?". World Bank. Policy Research Working Paper, $\mathrm{n}^{\circ}$ 3341. Washington

CHEN, SHAOHUA Y RAVALLION, MARTIN (2007). "Absolute Poverty Measures for the Developing World, 1981-2004". World Bank. Policy Research Working Paper, $\mathrm{n}^{\circ}$ 4211. Washington

CHEN, SHAOHUA Y RAVALLION, MARTIN (2012). An update to the World Bank's estimates of consumption poverty in the developing world. http://siteresources.worldbank.org/INTPOVCALNET/Resources/Global_Pove rty_Update_2012_02-29-12.pdf

CHENERY, HOLLIS (1974). Redistribution with Growth: Policies to Improve Income Distribution in Developing Countries in the Context of Economic Growth. Oxford University Press.

CLEMENS, MICHAEL A., KENNY, CHARLES J., Y MOSS, TODD J. (2007). "The trouble with the MDGs: confronting expectations of aid and development success" en World Development, vol.35, n 5, pp.735-751. 
COLLIER, PAUL Y DOLLAR, DAVID (2000). "Aid Allocation and Poverty Reduction". World Bank Policy Research Working papers, $n^{\circ}$ 2403. Washington.

COLLIER, PAUL Y DOLLAR, DAVID (2001) Development Effectiveness: What have we learnt? http://www.oecd.org/dataoecd/16/2/2664872.pdf)

COMISIÓN INDEPENDIENTE SOBRE PROBLEMAS INTERNACIONALES DEL DESARROLLO (Comisión Brandt) (1981). Dialogo Norte-Sur. México: Nueva Sociedad.

DATT, GAURAV, Y RAVALLION, MARTIN (2002). "Is India's Economic Growth Leavingthe Poor Behind?". World Bank. Policy Research Working Paper, $\mathrm{n}^{\circ}$ 2846. Washington

DEATON, ANGUS (2003). "Health, Inequality and Economic Development" en Journal of Economic Literature, Vol. 41, No. 1, pp. 113-158.

DEVARAJAN, SHANTAYANAN, MILLER, MARGARET J., Y SWANSON, ERIC V. (2002). "Goals for development: history, prospects and costs". World Bank Policy Research Working Paper, ${ }^{\circ} 2819$. Washington.

DOLLAR, DAVID (2006). Globalización, Desigualdad y Pobreza a partir de 1980. Libertad Desarrollo. Serie Informe Social no 102.

EASTERLY, WILLIAM (2006). The white man's burden: why the west's efforts to aid the rest have done so much ill and so little good. New York: Pinguin.

EASTERLY, WILLIAM (2008). Reinventing Foreing Aid. Cambridge, Massachusetts: MIT Press.

EMMERIJ, LOUIS; JOLLY, RICHARD; Y WEISS, THOMAS G. (2001). Ahead of the curve? UN Ideas and Global Change. Bloomington: Indiana University Press-United Nations Intellectual History Project.

FUJUYAMA, FRANCIS (2012). "The Future of History", en Foreign Affairs no 91.

HANSEN, HENRIK Y TARP, FINN (2001). "Aid and Growth Regressions" en Journal of Development Economics, $n^{\circ}$ 64, pp. 547-70.

JOLLY, RICHARD (2005). Global Inequality in historical perspective. UNUWIDER Jubilee Conference. http://www.rrojasdatabank.info/widerconf/ Jolly.pdf.

KABEER, NAILA (2010). Can the MDGs provide a pathway to social justice? The challenge of intersecting inequalities. Institute of Development Studies and MDG Achievement Fund.

KAKWANI, NANAK (1986). Analyzing redistribution policies: a study using Australian data. New York: Cambridge University Press.

KANBUR, RAVI (2010). "Globalization, Growth and Distribution: faming the questions", en Ravi Kanbur y Michael Spencer (eds.). Equity and Growth in a Globalizing World. Commission on Growth and Development. Washington: World Bank.

MILANOVIC, BRANKO (2005). Worlds apart: Measuring International and Global Inequality. Princeton University Press. 
MORAWETZ, DAVID (1977). Twenty-five years of Economic Development, 1950-1975. The John Hopkins University Press.

NACIONES UNIDAS (1974). "Declaración sobre el establecimiento de un Nuevo Orden Económico Internacional". Nueva Sociedad, n 14, pp. 50-53.

PNUD (1999). Informe sobre el Desarrollo Humano 1999. N. York.

PNUD (2003). Informe sobre el Desarrollo Humano 2003. N. York.

PNUD (2005). Informe sobre el Desarrollo Humano 2005. N. York.

RAVALLION, MARTIN (2000). "Should Poverty Measures Be Anchored to the National Accounts?" en Economic and Political Weekly, $\mathrm{n}^{\circ} 34$, pp. 32453252.

RAVALLION, MARTIN; CHEN, SHAOHUA Y SANGRAULA, PREM (2008). "Dolar a day revisited". World Bank Policy Research Working Paper, $\mathrm{n}^{\circ}$ 4620. Washington.

REDDY, SANJA Y POGGE THOMAS (2002). How Not to Count the Poor! A Reply to Ravallion. http://www.columbia.edu/ sr793/poggereddyreply.pdf.

RIDDELL, ROGER C. (2007). Does Foreign Aid Really Work? New York: Oxford University Press.

SACHS, JEFFREY (2005). The end of poverty: Economic possibilities for our time. New York: Penguin Press.

SACHS, JEFFREY Y WARNER, ANDREW (1995). "Economic convergence and economic policies". National Bureau of Economic Research Working Paper, 5039. Cambridge, MA.

SALA I MARTIN, XAVIER (2001). "The World Distribution of Income Estimated from Individual Country Distribution". National Bureau of Economic Research, $\mathrm{n}^{\circ}$ 8933. Cambridge, Massachusetts.

SCHABBEL, CHRISTIAN (2007). The Value Chain of Foreign Aid. Development, Poverty Reduction and Regional Conditions. New York: Physica-Verlag.

SELIGSON, MITCHELL A. (Ed.) (1984). The gap between rich and poor. Westview Press. Boulder.

SEN, AMARTYA (1983). "Poor, relatively speaking" en Oxford Economic Papers, $n^{\circ} 35$, pp. 153-169.

SEVERINO, JEAN-MICHEL Y RAY, OLIVER (2009). "The End of ODA: Death and Rebirth or a Global Public Policy". Center for Global Development Working Paper, no 167 (March).

SOBHAN, REHMAN (2006). "Challenging the injustice of poverty: rethinking aid strategies" en Steen Folke and Henrik Nielsen (Eds.). Aid impact and poverty reduction. New York: Palgrave-Macmillan.

SÖRENSEN, JENS S. (2010). Challenging the Aid Paradigm. Western Currents and Asian Alternatives. London: Palgrave Macmillan.

SOTILLO, JOSÉ ÁNGEL (2011). El sistema de cooperación para el desarrollo. Madrid: IUDC- Catarata. 
STEWART, FRANCES (1985). Basic Needs in Developing Countries. Baltimore: The Johns Hopkins University Press.

STEWART, FRANCES, SAITH, RUHI, Y HARRIS-WHITE, BARBARA (Eds.) (2007). Defining poverty in the developing world. New York: Palgrave-Macmillan.

STIGLITZ, JOSEPH (2006). Cómo hacer que funcione la globalización. Madrid: Taurus.

STREETEN, PAUL, ET AL. (1981). First Things First. New York: Oxford University Press.

SUTCLIFFE, BOB (2011). "La desigualdad global actualizada". Boletín de Recursos de Información, № 27, Instituto Hegoa.

UN MILLENNIUM PROJECT (2005). Investing in development: a practical plan to achieve the Millennium Development Goals. New York.

UNCETA, KOLDO (2007). "Globalización y pobreza: viejos y nuevos debates" en Javier Martínez-Peinado y Ramón Sánchez Tabarés: El futuro imposible del capitalismo. Ensayos en memoria de José María Vidal Villa. Barcelona: Icaria-Antrazyt.

UNCETA, KOLDO, GUTIÉRREZ-GOIRIA, JORGE Y AMIANO, IRATXE (2010). "Financing Development: ODA versus FDI and Remittances in the Most Vulnerable Countries" en Current Research Series, 9, pp. 165-186.

UNESCO (1970). El Informe Pearson. Una nueva estrategia para un desarrollo global. París: El Correo de la UNESCO.

VANDEMOORTELE, JAN (2002). Are we really reducing global poverty? United Nations Development Programme. Bureau for Development Policy.

VANDEMOORTELE, JAN (2009). "The MDG Conundrum: meeting the targets without missing the point" en Development Policy Review, $\mathrm{n}^{\circ} 27$ (4), pp. 355371.

VAN DER VEEN, A. MAURITS (2011). Ideas, Interests and Foreign Aid. Cambridge: Cambridge University Press.

WORLD BANK (1990). World Development Report 1990. Washington.

WORLD BANK (1998). Assessing Aid: What Works, What Doesn't, and Why. Oxford University Press.

WORLD BANK (2000). World Development Report 2000-2001. Washington.

WORLD BANK (2002). A Case for AID. Building a Consensus for Development Assistance. Washington.

WORLD BANK (2012a). World Development Indicators (on-line).

WORLD BANK (2012b). PovcalNet: the on-line tool for poverty measurement developed by the Development Research Group of the World Bank. 
